\title{
Método sensorless para PMSM basado en la derivada de la corriente para muy bajas velocidades
}

\author{
Alberto Sanz, Estanis Oyarbide \\ Group of Power Electronics and Microelectronics (GEPM) \\ Instituto de Investigación en Ingeniería de Aragón (I3A) \\ Universidad de Zaragoza, Mariano Esquillor s/n, 50018, Zaragoza, Spain. \\ Tel. +34-976762707, e-mail: albertosanz@unizar.es
}

\section{Resumen}

Se presenta un estimador de la posición del rotor basado en la derivada de la corriente para un motor síncrono de imanes permanentes (SPMSM). El objetivo es minimizar la influencia en la estimación de las incertidumbres en los parámetros de la máquina y de errores en las medidas.

\section{Introducción}

De forma general hay dos grupos de técnicas de estimación de la posición, las basadas en modelos fundamentales de la máquina y las basadas en los modelos anisótropos. Las primeras, se basan en la estimación del flujo o fuerza contra-electromotriz (back-EMF) para determinar la posición, [1]. Estas técnicas se basan en la integración para obtener el flujo, por lo que, errores en la medida, incertidumbres del modelo de la máquina o las no linealidades del inversor inciden fuertemente en la estimación. La back-EMF disminuye con la velocidad siendo despreciable a muy bajas velocidades y nula en parado. Por lo tanto, estos métodos no son aplicables en parado y para bajas velocidades presentan una gran dependencia a las incertidumbres en los parámetros de la máquina.

Las basadas en modelos anisótropos explotan las asimetrías de la máquina. De esta manera, tienen una menor dependencia que las anteriores frente a variaciones de los parámetros de la máquina y presentan buenas prestaciones en parado y en bajas velocidades, pero generalmente requieren inyectar señales adicionales, [2], incrementando el rizado del par, la implementación en tiempo real es más compleja y pierden en eficiencia y en dinámica cuando la velocidad aumenta. A demás, el SPMSM suele presentar una mayor dificultad para aplicar técnicas basadas en asimetrías respecto del motor síncrono de ímanes insertados (IPMSM), puesto que no hay diferencia entre las inductancias del eje directo y cuadratura, $\mathrm{L}_{\mathrm{d}}=\mathrm{L}_{\mathrm{q}}$, frente a la máquina de imanes insertados, $\mathrm{L}_{\mathrm{d}}<\mathrm{L}_{\mathrm{q}}$.
Consecuentemente, han surgido métodos que no inyectan señales basados en la derivada de la corriente, [3], para IPMSM. Son robustos a muy bajas velocidades al no estar basados en la integración y no trabajar con valores medios de tensión. Pero sólo son aplicables al IPMSM y realizan la medida del di/dt en el intervalo de conmutación del vector cero-tensión. En la mayoría de los casos, el di/dt en el vector cero-tensión, ver Fig. 1, es despreciable, lo que dificulta su aplicación.

Se ha desarrollado un método de identificación de la posición para bajas velocidades basado en la derivada de la corriente tanto para IPMSM como SPMSM sin estar restringida la realización de la medida del di/dt al vector cero-tensión.

\section{Identificación de la posición}

\section{Parado}

La aplicación en la que se va a utilizar el SPMSM no permite previamente posicionar el rotor en una posición conocida. Estando el SPMSM parado no es posible aplicar el estimador basado en el $\mathrm{di} / \mathrm{dt}$, por lo que se utiliza una estrategia basada en la saturación de las inductancias. Aplicando una excitación suficiente, alineada con el flujo del imán se consigue una saturación máxima y por lo tanto un valor mínimo de inducción. Utilizando esta propiedad, se realiza un barrido de vectores de tensión en el SPMSM y analizando su respuesta en corriente se obtiene la posición.

\section{Bajas velocidades}

A partir de las ecuaciones de la máquina síncrona de imanes permanentes en los ejes estacionarios $\alpha,(1)$, y $\beta$, (2), donde $\emptyset_{\mathrm{m}}$ es el flujo de los imanes, $\mathrm{L}_{\alpha}$ es la inductancia en el eje $\alpha, L_{\beta}$ es la inductancia en el eje $\beta$ y $L_{\alpha \beta}$ es la inductancia mutua, se obtiene una ecuación con la posición del rotor en función de la derivada de la corriente. 


$$
\begin{gathered}
V_{\alpha}=R_{s} i_{\alpha}+\frac{d}{d t}\left(L_{\alpha} i_{\alpha}\right)+\frac{d}{d t}\left(L_{\alpha \beta} i_{\beta}\right) \\
+\frac{d}{d t}\left(\emptyset_{m} \cos \theta\right) \\
V_{\beta}=R_{s} i_{\beta}+\frac{d}{d t}\left(L_{\beta} i_{\beta}\right)+\frac{d}{d t}\left(L_{\alpha \beta} i_{\alpha}\right) \\
+\frac{d}{d t}\left(\emptyset_{m} \operatorname{sen} \theta\right)
\end{gathered}
$$

El estimador recibe los valores instantáneos de tensión en los devanados del estator $\left(2 / 3 \mathrm{~V}_{\mathrm{BUS}}\right.$, $1 / 3 \mathrm{~V}_{\mathrm{BUS}}$ ), frente a los valores medios con los que se trabaja en el resto de estrategias. Para muy bajas velocidades los valores de tensión medios en las fases son muy bajos, por lo que, desviaciones pequeñas en la estimación de la resistencia o de las tensiones de fase generan estimaciones erróneas en la posición.

En la literatura se realiza la medida del di/dt en el intervalo de conmutación del vector cero-tensión, ver Fig. 1, ya que: a) de esta forma, se puede eliminar un término de la ecuación empleada asociado a las tensiones $\mathrm{V}_{\alpha}$ y $\mathrm{V}_{\beta}, \quad$ b) en la modulación vectorial estándar, el vector cerotensión es el de mayor duración para bajas velocidades, facilitando una medida precisa. Pero en la mayoría de los casos, el di/dt en el vector cerotensión es despreciable, lo que dificulta la aplicación de la estrategia descrita.

Para poder realizar una medida precisa del di/dt en los vectores activos, se ha desarrollado una modulación vectorial novedosa que permite incrementar el tiempo de los vectores activos, resultando en un compromiso entre el tiempo mínimo para realizar la medida y el rizado de la corriente.

\section{Resultados de simulación}

Se ha implementado en matlab-simulink la estrategia sensorless basada en el $\mathrm{di} / \mathrm{dt}$ anteriormente descrita con el objetivo de validar su robustez en simulación. Se han realizado simulaciones para distintas velocidades, pares y errores en la estimación de los parámetros del SPMSM bajo estudio. En la Fig. 2 se muestra una estimación de la posición suponiendo una desviación en la estimación de la resistencia de fase sobre la real del 50\% para 5rpm. Se puede apreciar que la incidencia de una mala estimación de la resistencia de fase a muy bajas velocidades es casi despreciable, siendo en la mayoría de las estrategias sensorless determinante.

\section{Conclusiones}

En la literatura, los métodos sensorless basados en la derivada de la corriente sólo se aplican a IPMSM y la estimación se realiza únicamente en el vector cero. Se ha desarrollado un método de identificación de la posición basado en la derivada de la corriente tanto para IPMSM como para SPMSM, pudiéndose realizar la estimación de la posición en los vectores activos. De esta manera, este método se puede utilizar en cualquier PMSM.

\section{REFERENCIAS}

[1]. BOLDEA, I., PAICU, M.C., ANDREESCU, G.-D., and BLAABJERG, F. Active Flux' DTFC-SVM Sensorless Control of IPMSM. IEEE Transactions Energy Conversion. 2009, vol. 24, no. 2, pp. 314-322.

[2]. YUAN, T. and DOKI, S. An experimental study of position sensorless control at low speed of IPMSM with heavy magnetic saturation. 40th Annual Conference of the IEEE Industrial Electronics Society. Dallas: IECON, 2014, pp. 398-403.

[3]. MAKAINO, Y., SONG, H. and DOKI, S. A position sensorless control for IPMSM at low speed based on current response at zero voltage vector without any additional signal injection. 2016 IEEE Symposium on Sensorless Control for Electrical Drives (SLED). Nadi: SLED, 2016, pp. 1-4. 


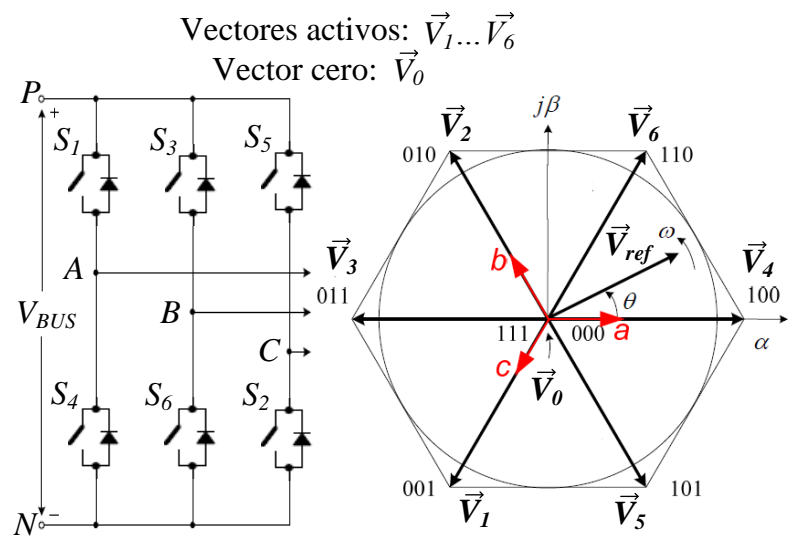

Fig. 1. Modulación vectorial.

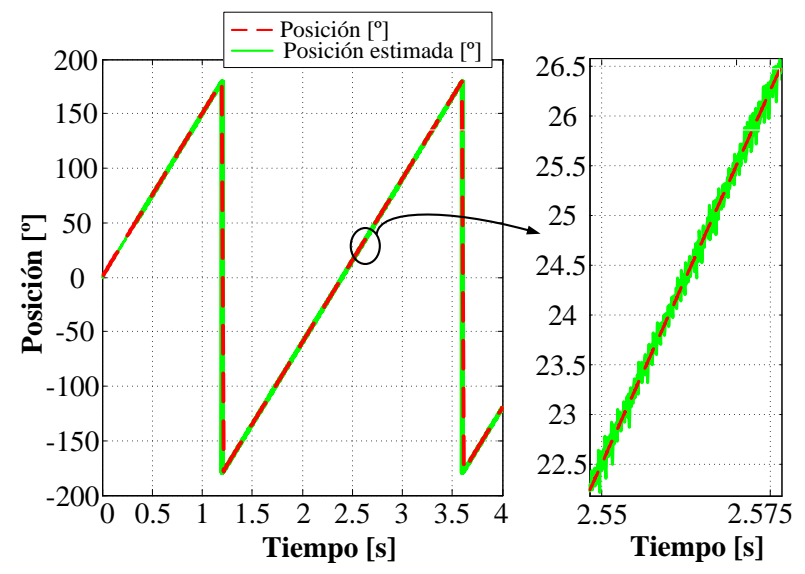

Fig. 2. Estimación posición para 5rpm y $115 \mathrm{Nm}$. 